doi:10.17659/01.2019.0010

Journal of Case Reports 2019;9(1):36-39

\title{
Ado-trastuzumab Emtansine-Associated Spider Naevi in Metastatic HER2- positive Breast Cancer
}

\author{
Karla A. Lee ${ }^{1}$, Scott Shepherd ${ }^{1}$, Nicolo Battisti ${ }^{1}$, Alicia Okines ${ }^{1}$, Mark Allen ${ }^{1}$, Louise Fearfield ${ }^{2}$ \\ Departments of ${ }^{1}$ Breast Oncology and ${ }^{2}$ Dermatology, The Royal Marsden NHS Foundation Trust, London.
}

\section{Corresponding Author: \\ Dr. Karla Lee \\ Email: Karla.lee@nhs.net}

This is an Open Access article distributed under the terms of the Creative Commons Attribution License (creativecommons.org/ licenses/by/3.0).

Received : November 1, 2018

Accepted : January 28, 2019

Published : February 20, 2019

\begin{abstract}
Background: Ado-trastuzumab emtansine (T-DM1), has improved rates of progressionfree and overall survival amongst patients treated for human epidermal growth factor receptor 2 (HER2)-positive metastatic breast cancer (mBC). The development of spider naevi/telangiectasia represents an under-reported adverse event amongst patients being treated with T-DM1. The mechanism of development of T-DM1 associated telangiectasia is unknown. Case Report: Here, we report the case of a 53 year old female patient with metastatic HER2 positive breast cancer. Following 12 months of treatment with T-DM1, she developed many tens of spider naevi across her face, chest, and arms. She was not thrombocytopaenic, nor did her biochemistry reveal a transaminitis. She did have an elevated bilirubin, notwithstanding her history of Gilbert's Syndrome. At the time these skin lesions appeared, she was also suffering from poly-arthralgia, presumed to be toxicity of previous anti-cancer immunotherapy. She was given a three-week course of reducing steroid, and experienced a significant reduction in the number of spider naevi. For cosmetic purposes, she has undergone a session of hyfrecation to a number of remaining lesions, and will be seen with a view to further hyfrecation in a number of months. Conclusion: The development of telangiectasia represents a newly recognized adverse effect of T-DM1, and one of which dermatologists and oncologists alike, should be aware. Oncologists should actively enquire about, and examine for, these lesions, while dermatologists should be aware that the pattern of mucocutaneous lesions can mimic hereditary haemorrhagic telangiectasia (HHT), present a bleeding risk, and produce cosmetic concerns for patients.
\end{abstract}

Keywords: Ado-trastuzumab Emtansine, Breast Neoplasms, Oncologists, Receptor, Telangiectasia.

\section{Introduction}

Ado-trastuzumab emtansine (T-DM1), has improved median progression-free and overall survival amongst patients treated for HER2positive metastatic breast cancer $(\mathrm{mBC})[1,2]$. Here we present a case of a patient developing tens of spider naevi whilst receiving treatment with T-DM1, and review the medical literature.

\section{Case Report}

The patient was diagnosed with a left-sided HER2positive breast cancer in 2010. She had no significant medical, family or medication history. Following surgery, chemotherapy, radiotherapy and one year of adjuvant Trastuzumab, she unfortunately relapsed in her left supra-clavicular fossa. She received 6 cycles of Docetaxel, Trastuzumab and Pertuzumab, followed by consolidation radiotherapy and maintenance Trastuzumab and Pertuzumab until May 2017. At that time, a new cerebellar metastasis and right axillary nodal relapse were diagnosed, and following radiotherapy to her posterior fossa, she enrolled in a trial of T-DM1 and Atezolizumab (a monoclonal antibody against the protein programmed cell death-ligand 1) or 
placebo. Due to presumed immunotherapy toxicity, she was unblinded and it was confirmed that she had been randomized to the immunotherapy arm. Following a short treatment break she resumed T-DM1 alone and continues on this.

In July of 2018, twelve months following initiation of T-DM1 therapy, the patient reported the development of many tens of spider naevi across her face, chest, and arms, which appeared over a period of four weeks [Fig.1]. She had not experienced any bleeding but was concerned by her cosmetic appearance. Blood tests showed no thrombocytopenia or transaminitis. The bilirubin was elevated, not withstanding her history of Gilbert's syndrome. At the time of appearance of these skin lesions, she was also suffering from poly-arthralgia, presumed to be an immunotherapy toxicity. She was given a three-week course of reducing steroid for this poly-arthralgia, and experienced a significant reduction in the number of spider naevi. This response has been sustained to date. She has undergone a session of hyfrecation for a number of remaining lesions and will be seen with a view to further hyfrecation in a number of months.

\section{Discussion}

T-DM1 is an antibody-drug conjugate composed of Trastuzumab, a thioether linker, and a microtubule inhibitor, DM1 (Emtansine), and it is typically utilized for advanced HER2-positive breast cancer for patients who relapse within six months of completing adjuvant Trastuzumab therapy or whose disease progresses on an anti-HER2 agentcontaining regimen [3]. T-DM1 exerts a two-hit effect by disrupting HER2 signaling, and delivering a cytotoxic agent directly to HER 2 over-expressing cells.

The large phase III EMILIA trial compared T-DM1 to combined Lapatinib and Capecitabine [2]. The most common serious toxicities associated with T-DM1 were thrombocytopenia (13\%)

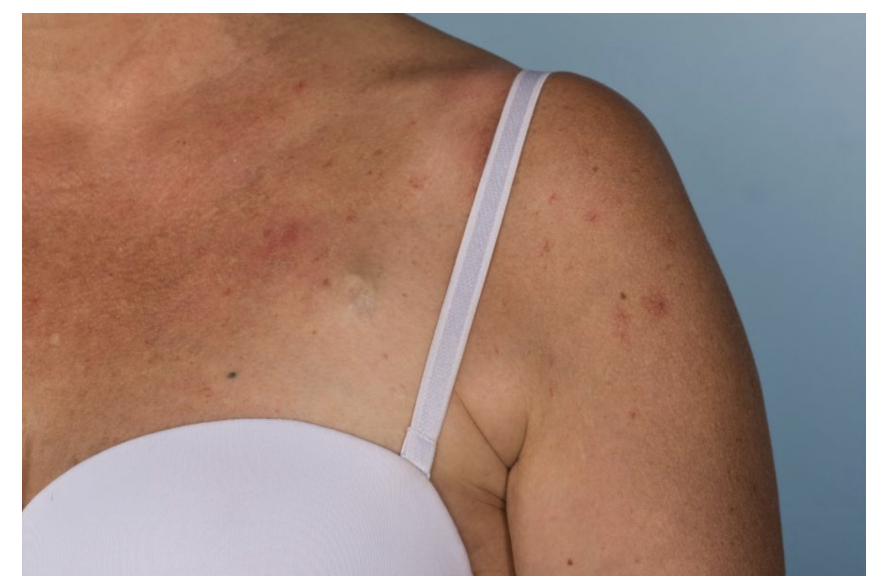

Fig.1: Spider naevi on chest and arm.

and elevated liver function enzymes: aspartate aminotransferase and alanine aminotransferase (7\%). Nodular regenerative hyperplasia is a rare complication of treatment with T-DM1 and can cause portal hypertension and decompensated liver failure [4]. Also, patients treated with T-DM1 in the EMILIA trial experienced an overall higher rate of bleeding compared with those treated with Capecitabine plus Lapatinib (30 versus 16 percent, respectively), though the rate of serious bleeding events was low in both arms ( 1.4 versus 0.8 percent). However, the etiology of bleeding is not entirely explained by other risk factors (e.g., concomitant T-DM1-related thrombocytopenia). For example, in this trial, one patient who received T-DM1 experienced a grade 4 gastrointestinal bleeding event despite having a normal platelet count. Furthermore, cases of intra-cranial hemorrhage $[5,6]$ and expansion of radiation necrosis due to telangiectasia and hemorrhage [7] have been reported. Based on these findings, the FDA revised the label for T-DM1 in July 2014 to highlight this increased risk of bleeding with recommendations for careful follow-up for patients during treatment with T-DM1 [8].

The development of spider naevi/ telangiectasia represents an under-reported adverse event, and may be part of the same phenomenon as the unexplained bleeding associated with T-DM1. 
Sibaud et al. reported five patients in a case series [9], and then a year later published an additional five cases, discussing the high bleeding risk in this cohort [10]. The only other published literature is a case report describing a patient experiencing hereditary hemorrhagic telangiectasia (HHT)like symptoms of spider-angiomata lesions, epistaxis, and hematochezia, which resolved with discontinuation of T-DM1 [8].

Spider naevi represent a distinct type of telangiectasia characterized by a central arteriole surrounded by multiple small, radiating dilated vessels. The mechanism of development of T-DM1 associated mucocutaneous telangiectasia is unknown. The development of telangiectasia has been associated with other drugs including calcium channel blockers and lithium, and most obviously with chronic decompensated liver disease. In the case series by Sibaud et al., mild elevation in transaminases was observed in all five reported patients [9]. Of note, one of the major adverse effects of T-DM1 is liver injury with associated elevations in transaminases [1]. Alternatively, a role for disruption of endothelial microtubules by Emtansine in T-DM1-associated telangiectasia has been suggested [9], as this also seems to be a factor in the pathological basis of HHT syndrome, and cytoskeletal rearrangement which plays an important role in arteriole dilatation $[11,12]$. Skin toxicity is rare with Trastuzumab treatment, due to low HER2 expression in the epidermis [13], but it has been suggested that HER2 expression on endothelial cells could facilitate the Trastuzumabmediated delivery of Emtansine directly to these cells, which could cause the disruption of microtubules, impairing angiogenesis and leading to the development of spider naevi [9].

Recognition and treatment of drug toxicities is critical for the safety profile of anticancer therapies, in order to improve outcomes by preventing treatment discontinuation for reasons not related to disease progression.

\section{Conclusion}

The development of telangiectasia represents a newly recognized adverse effect of T-DM1, and one of which dermatologists and oncologists alike, should be aware. Oncologists should actively enquire about, and examine for, these lesions, while dermatologists should be aware that the pattern of mucocutaneous lesions can mimic HHT, present a bleeding risk, and produce cosmetic concerns for patients. Steroids may lead to reduction in number of lesions and can be considered as a treatment option.

Contributors: KL: manuscript writing, patient management; $\mathrm{SS}, \mathrm{NB}, \mathrm{AO}, \mathrm{MA}$ : manuscript editing, patient management; LF: critical inputs into the manuscript. KL will act as guarantor. All authors approved the final version of this manuscript.

Funding: None; Competing interests: None stated.

\section{References}

1. Verma S, Miles D, Gianni L, Krop IE, Welslau M, Baselga J, et al. Trastuzumab emtansine for HER2positive advanced breast cancer. $\mathrm{N}$ Engl $\mathrm{J}$ Med. 2012;367:1783-1791.

2. Dieras V, Miles D, Verma S, Pegram M, Welslau $\mathrm{M}$, Baselga $\mathrm{J}$, et al. Trastuzumab emtansine versus capecitabine plus lapatinib in patients with previously treated HER2-positive advanced breast cancer (EMILIA): a descriptive analysis of final overall survival results from a randomised, open-label, phase 3 trial. The Lancet Oncology. 2017;18(6):732-742.

3. Isakoff SJ, Baselga J. Trastuzumab-DM1: building a chemotherapy-free road in the treatment of human epidermal growth factor receptor 2-positive breast cancer. J Clin Oncol. 2011;29(4):351-354.

4. Force J, Saxena R, Schneider BP, Storniolo AM, Sledge GW, Chalasani N, et al. Nodular regenerative hyperplasia after treatment with Trastuzumab Emtansine. J Clin Oncol. 2016;34(3):e9-e12.

5. Kolarich AR, Reynolds BA, Heldermon CD. Adotrastuzamab emtansine associated hyponatremia and intracranial hemorrhage. Acta Oncologica. 2014;53(10):1434-1436.

6. Okines A, Irfan T, Khabra K, Smith I, O’Brien M, Parton M. Development and responses of brain metastases during treatment with trastuzumab emtansine (T-DM1) for HER2 positive advanced breast cancer: A single institution experience. Breast J. 2018;24(3):253-259. 
7. Mitsuya K, Watanabe J, Nakasu Y, Hayashi N, Harada $H$. Expansive hematoma in delayed cerebral radiation necrosis in patients treated withT-DM1: a report of two cases. BMC Cancer. 2016:16 (391).

8. Kwon Y, Gomberg-Maitland M, Pritzker M, Thenappan $\mathrm{T}$. Telangiectasia and pulmonary arterial hypertension following treatment with Trastuzumab Emtansine: A Case Report. Chest. 2016;149(4):103-105.

9. Sibaud V, Niec RE, Schindler K, Busam KJ, Roche H, Modi S, Delord JP, Lacouture ME, et al. Ado-trastuzumab emtansine-associated telangiectasias in metastatic breast cancer: a case series. Breast Cancer Res. 2014;146:451456.

10. Sibaud V, Vigarios E, Combemale P, Lamant $P$, Lacouture ME, et al. T-DM1-related telangiectasias: a potential role in secondary bleeding events. Annals of Oncology. 2015;26(2):436-437.

11. Loufrani L, Henrion D. Role of the cytoskeleton in flow (shear stress)-induced dilation and remodeling in resistance arteries. Med Biol Eng Comput. 2008;46:451460.

12. Fernandez-LA, Sanz-Rodriguez F, Blanco FJ, Bernabéu C, Botella LM, et al. Hereditary hemorrhagic telangiectasia, a vascular dysplasia affecting the TGFsignaling pathway. Clin Med \& Res. 2006;4:66-78.

13. Krahn G, Leiter U, Kaskel P, Udart M, Utikal J, Bezold $\mathrm{G}$, et al. Coexpression patterns of EGFR, HER2, HER3 and HER4 in non-melanoma skin cancer. Eur J Cancer. 2001;37:251-259. 